\title{
NIVEL DE SATISFACCIÓN DE LOS ESTUDIANTES DE LA FACULTAD DE ENFERMERÍA DE LA UNCP EN RELACIÓN AL PROCESO DE FORMACIÓN PROFESIONAL- $2009^{37}$
}

\author{
Yallico Madge Luz Consuelo 1, Quispe Condori Nelly Flora 2, Huachos Benites Marco 3 \\ Facultad de Enfermería de la Universidad Nacional del Centro del Perú
}

\begin{abstract}
RESUMEN
La Satisfacción del Estudiante refleja la eficiencia de los diversos aspectos educativos Institucionales. Suministrar información sobre la gestión institucional basada en la perspectiva de los estudiantes, orienta la toma de decisiones y provee información de las condiciones del entorno académico y administrativo que impactan en la percepción de satisfacción 0 insatisfacción con el proceso de formación que la Facultad brinda a los estudiantes. En este contexto la investigación tiene como objetivo: determinar el nivel de satisfacción de los estudiantes de la Facultad de Enfermería de la UNCP en relación a su formación profesional. La población estuvo conformada por los estudiantes de la facultad de Enfermería y la muestra fue constituída por 72 estudiantes distribuidos equitativamente entre el II y IX semestre. La técnica de recolección de datos fue la encuesta. El estudio evidencial: el nivel de satisfacción de los estudiantes en relación al proceso de formación profesional està directamente relacionado con las condicionantes académicas, administrativas y servicios que brinda la Institución formadora. El grado de eficacia de los diferentes servicios que brinda la Facultad es regular en su mayoría. La satisfacción de los estudiantes de Enfermería con respecto a su formación de pre grado, es positiva a pesar de evidenciar su disconformidad por la atención administrativa. La formación de los estudiantes es positiva con una tendencia de regular a buena. Existe una relación directa entre el nivel de satisfacción y la formación profesional.
\end{abstract}

Palabras Clave: Nivel de satisfacción, satisfacción de estudiantes, formación profesional, calidad

\section{STUDENTS SATISFACTION LEVEL OF THE UNCP NURSING FACULTY REGARDING THE PROCESS OF VOCATIONAL TRAINING -} 2009

\begin{abstract}
The student Satisfaction reflects the efficiency of various institutional aspects of education. Provide information on institutional management based on the students perspective, guide decision making and provides information on the conditions of academic and administrative environment that impact the perception of satisfaction or dissatisfaction with the process of training the College provides students. In this context, the research aims: to determine the students satisfaction level of students in the School of Nursing at UNCP in relation to vocational training. The population was made up of students of the College of Nursing and the sample consisted of 72 students distributed evenly between the second and ninth semester. The collection technique was the survey data. The study shows: That the student satisfaction level regarding the training process is directly related to academic constraints, administrative and services provided by the institution that forms. The degree of effectiveness of the various services offered by the Faculty is mostly regular. The satisfaction of nursing students about their undergraduate training, is positive despite the evident discomfort over administrative attention. The training of students is positive with a tendency fair to good. There is a direct relationship between the level of satisfaction and professional training.
\end{abstract}

Key Words: Level of satisfaction, student satisfaction, vocational training, quality

37 Trabajo de investigación fue recibido el 07/05/2010 retornado para su revisión el 15/06/2010 y aprobado para su publicación 16/11/2010. 


\section{INTRODUCCIÓN}

El punto de partida de toda gestión de calidad en procesos educativos, consiste en captar las exigencias de los estudiantes, docentes y egresados y analizar la forma de ofrecer soluciones que respondan a sus necesidades. En la evaluación de la efectividad lograda es importante el criterio de aquellos que reciben la influencia directa en el proceso docente educativo, es decir, los estudiantes como futuros graduados universitarios haciendo énfasis en su competencia profesional. La satisfacción de los estudiantes en una institución educativa y su continuidad en la carrera, evitando deserciones, son componentes esenciales para incrementar la competitividad de las organizaciones educativas.

La satisfacción de los estudiantes reflejan la eficiencia de los diversos aspectos educativos Institucionales. Suministrar información sobre la gestión institucional basada en la perspectiva de los estudiantes, tiene por finalidad orientar la toma de decisiones.

El reto de enfermería en este milenio no es solo continuar fortaleciendo la integración interna aisladamente, es también desarrollar y utilizar estrategias de trabajo articuladoras. La visión de que Enfermería asuma mayor responsabilidad en acciones de promoción y protección de la salud y una orientación más preventiva fue y es en la actualidad uno de los roles básicos para el profesional de Enfermería por lo que se hace necesario conocer el nivel de satisfacción de los estudiantes de la facultad de Enfermería de la UNCP en relación al 'proceso de formación profesional 2009

La transformación en la educación de enfermería requiere de nuevos procesos para enfocar la formación desde una perspectiva más integra a través de la interdisciplinariedad, que a su vez necesita de cambios en la estructura organizativa de los programas de formación, orientación del currículo a fin de lograr la tan ansiada Acreditación universitaria que permita ofrecer a la comunidad profesionales altamente competitivos garantizando una atención hacia el cuidado de la persona.

En el contexto del proceso de Acreditación, es importante contar con indicadores que nos aproximen al conocimiento de las condiciones del entorno Académico y administrativo que impactan en la percepción de satisfacción o no satisfacción con el proceso de formación que la facultad de Enfermería brinda a los estudiantes, para la implementación de los planes de mejora en el marco de la Planificación estratégica, por lo que planteamos la siguiente interrogante: ¿Cuál es el nivel de satisfacción de los estudiantes de la Facultad de Enfermería en relación a su formación Profesional 2009?. La investigación se realizó con el objetivo de: Determinar el nivel de satisfacción de los estudiantes de la Facultad de Enfermería de la UNCP en relación a su formación Profesional.

El desarrollo alcanzado del término "satisfacción" en las instituciones educativas y la necesidad de evaluar la calidad de la docencia han hecho necesario asumir este concepto como uno de los indicadores de calidad en la prestación de los servicios. Confirmando esto, Laferriere (1), quien sostiene que la satisfacción del cliente constituye un indicador de calidad. Sea cual fuere la idea de una institución de calidad, deben ser observables determinados productos: satisfacción de usuarios, empleados, impacto claramente positivo en la sociedad y resultados económicos para mejorar la organización de los servicios a proveer.

En el caso de la enseñanza superior "se debe ver al estudiante como un consumidor a quién hay que servir", (Ivancevich e Ivancevich). Por ello, constituyen una importante fuente de información para la evaluación continua de cualquier institución educativa. Los datos que aportan permiten inferir características claves del funcionamiento de ella, determinando la medida en la que se alcanzan los fines preestablecidos.

La satisfacción del estudiante refleja la eficiencia de los diversos aspectos que componen el día a día de su experiencia educacional. La meta de la institución debe ser que los estudiantes que cursan una carrera manifiesten su satisfacción con el rigor de los cursos, con las interacciones con su profesor y los otros estudiantes, con la justicia con que se le evalúa, etc., así como con las instalaciones y equipamiento que apoyan dichas interacciones

A la Carrera de Enfermería, de la Universidad Nacional del Centro del Perú como una entidad al servicio de la sociedad le corresponde responder a las necesidades de sus clientes, desarrollando servicios atractivos que aseguren, además, su posicionamiento a nivel país. Sin embargo, adolece de una evaluación sistemática de mejora continua de la calidad de la docencia que permita identificar y fortalecer las conexiones entre la satisfacción del estudiante con los servicios prestados como Institución de Educación Superior. Se agrega a esto que, a través de conversaciones informales, por observaciones empíricas y el análisis de los programas de asignaturas, se ha evidenciado ciertas deficiencias y problemas, tanto en el ámbito académico como administrativo, que se relacionan directamente con el proceso de enseñanza-aprendizaje.

\section{MATERIALES Y MÉTODOS}

El estudio corresponde a una investigación de tipo descriptivo, ya que está orientada a describir las condicionantes académicas administrativas que influyen en el nivel de satisfacción de los estudiantes de Enfermería, mediante una encuesta en base a un grupo de indicadores que desde la óptica estudiantil nos da una visión amplia de 
la satisfacción de expectativas en el proceso de formación y su competencia profesional una vez graduados. La investigación es retrospectiva. Su diseño corresponde al no experimental de corte transversal. La población fue de 242 estudiantes, la muestra estuvo constituída por 72 estudiantes distribuidos entre el II y IX semestre; se tomó esta decisión debido a que estos estudiantes se verán favorecidos en términos de ciclos faltantes por las decisiones de mejoramiento que adopten las autoridades; y porque tienen un conocimiento de las fortalezas y debilidades de la Universidad. Para el análisis de los resultados se utilizó los métodos de la estadística y la hoja de cálculo excel.

\section{RESULTADOS:}

Entre los datos generales de la muestra seleccionada, se tiene que el $81 \%$ tienen edades entre 16 y 20 años, un $18 \%$ tienen edades entre 23 y 25 años y solo el $1 \%$ tienen entre 26 a 30 años. Un buen porcentaje (95\%) pertenecen al sexo femenino. La mayoría (92\%) son solteras. El $78 \%$ provienen de Huancayo y alrededores y solo el $22 \%$ proviene de otros lugares. En relación a la Institución elegida y carrera que cursa, refieren que las razones principales son: El tener prestigio la UNCP como institución formadora estatal y su accesibilidad económica para los estudiantes

En relación a la percepción del usuario respecto a la atención recibida:

\section{Gráfico $\mathrm{N}^{\circ} 01$}

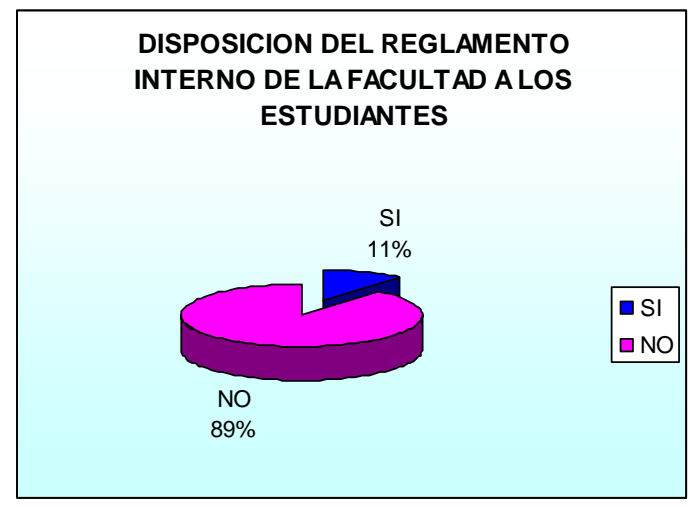

Fuente: Encuesta aplicada a estudiantes que conformaron la muestra.

A referencia de los estudiantes se tiene que un $89 \%$ manifiesta que no han tenido acceso al reglamento de la Facultad y solo un $11 \%$ de estudiantes sobre todo de los semestres avanzados tuvieron acceso a este documento a solicitud de ellos mismos.

\section{Gráfico $\mathrm{N}^{\circ} 04$}



Fuente: Encuesta aplicada a estudiantes que conformaron la muestra

En el presente gráfico se observa que los servicios tienen un grado de eficacia "regular" en su mayoría, pero llama la atención que los servicios del centro de cómputo de la facultad y del centro médico de la Universidad no son eficaces.

Tabla N ${ }^{\circ} 04$

TRATO, ATENCION Y ORIENTACION RECIBIDO EN LA FACULTAD

\begin{tabular}{lccc}
\hline $\begin{array}{c}\text { Trato, atención y orientación } \\
\text { recibida }\end{array}$ & Bueno & Regular & Malo \\
\hline Trato recibido en su permanencia en la & 10 & 58 & 04 \\
Facultad & 18 & 54 & 00 \\
Confianza por el personal docente para & 02 & 45 & 30 \\
expresar sus necesidades & 10 & 61 & 01 \\
Atención a las consultas por el & & & \\
personal administrativo & & & \\
Orientación recibida durante su \\
integración a la Facultad
\end{tabular}

Fuente: Encuesta aplicada a estudiantes que conformaron la muestra

\section{Gráfico No 08}




Fuente: Encuesta aplicada a estudiantes que conformaron la muestra.

En la tabla № 04 referente al trato, atención y orientación que reciben los estudiantes en la facultad, en su mayoría es "regular". El nivel de satisfacción con los servicios brindados por las diferentes estancias también se encuentra en ese nivel, pero llama la atención el servicio que brinda la fotocopiadora el cual es aceptado por un gran número de estudiantes encuestados como "buena"

\section{Gráfico $N^{0} 09$}



\section{Gráfico N ${ }^{\circ} 10$}

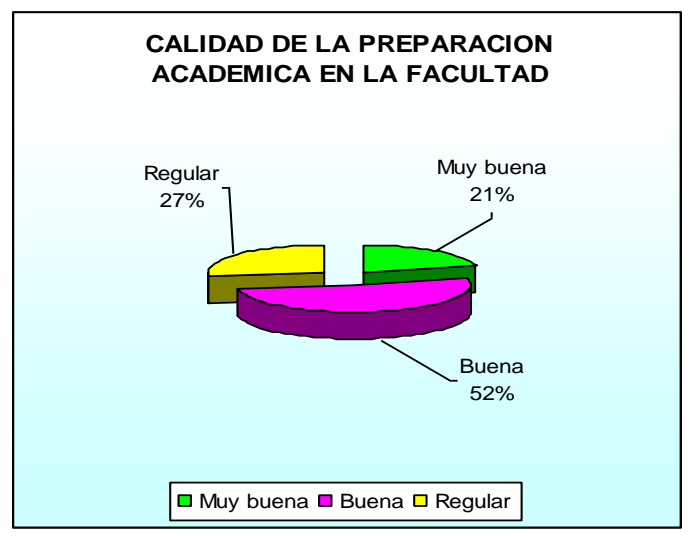

Fuente: Encuesta aplicada a estudiantes que conformaron la muestra

En el gráfico $\mathrm{N}^{0} 09$ se evidencia que los estudiantes encuestados están satisfechos con la profesión elegida, inclusive un buen porcentaje refiere estar muy satisfecho. En menor porcentaje hay estudiantes que se sienten poco satisfechos con la profesión.

En relación a la calidad de la formación Académica que se observa en el gráfico $N^{0} 10$, los estudiantes encuestados manifiestan que es buena (52\%), Regular (27\%), muy buena (21\%). También refieren que la formación social ha estimulado su desarrollo personal. Ellos recomiendan la urgente actualización del currículo 1995, así como los sílabos.

\section{DISCUSIÓN}

La satisfacción del estudiante refleja la eficiencia de los diversos aspectos que componen el día a día de su experiencia educacional. La meta de la institución debe ser que los estudiantes que cursan una carrera manifiesten su satisfacción académica, administrativa y social.

El grado de eficacia de los diferentes servicios que brinda la Facultad es "regular" en su mayoría a referencia de los encuestados. Pese a ello la satisfacción de los estudiantes de enfermería de la UNCP con respecto a su formación de pre grado, es positiva en lo académico, pero se evidencia disconformidad en la atención administrativa. El desarrollo alcanzado del término "satisfacción" en las instituciones educativas y la necesidad de evaluar la calidad de la docencia han hecho necesario asumir este concepto como uno de los indicadores de calidad en la prestación de los servicios (Laferriere, 1993).

Es importante en la educación superior considerar al estudiante como un consumidor a quien hay que servir, convirtiéndose en un indicador para la evaluación continua de la Institución Educativa. "La calidad en servicio consiste en satisfacer o sobrepasar las expectativas que tienen los clientes con respecto a usted o a su organización" (Cottle, D.1991)

El nivel de satisfacción del estudiante de pre grado de la facultad con los servicios brindados por las diferentes estancias es regular, pero el servicio que brinda la fotocopiadora es aceptado como "buena" por la mayoría de los estudiantes encuestados.

Más del $90 \%$ de los estudiantes encuestados evidencian satisfacción por su formación académica ya que las estrategias de enseñanza utilizada facilitan la investigación

A la Carrera de Enfermería, de la Universidad Nacional del Centro del Perú como una entidad al servicio de la sociedad le corresponde responder a las necesidades de sus clientes, desarrollando servicios atractivos que aseguren, además, su posicionamiento a nivel del país. Sin embargo, adolece de una evaluación sistemática de mejora continua de la calidad académica.

\section{CONCLUSIONES}

El nivel de satisfacción de los estudiantes en relación al proceso de formación profesional está directamente relacionado con las condicionantes académicas, 
administrativas y servicios que brinda la Institución formadora.

En lo académico, las autoridades facilitan los medios para un buen desempeño pero falta mayor apoyo en lo intelectual. En lo administrativo existe escasa información y accesibilidad a los documentos normativos.

El grado de eficacia de los diferentes servicios que brinda la Facultad es "regular", según referencia de la mayoría de los estudiantes encuestados.

La satisfacción de los estudiantes de enfermería de la UNCP con respecto a su formación de pre grado, es positiva a pesar de evidenciar su disconformidad por la atención en lo administrativo, indicando que existen aspectos a mejorar. La formación de los estudiantes es positiva con una tendencia de regular a buena.

Existe una relación directa entre el nivel de satisfacción y la formación profesional.

El nivel de satisfacción con los servicios brindados por las diferentes estancias también es regular, a diferencia del servicio que brinda la fotocopiadora, el cual es aceptado como "buena" a referencia de la mayoría de los estudiantes integrantes de la muestra.

\section{CONCLUSIONES}

- $\quad$ Por ser una profesión eminentemente social, amerita velar por la satisfacción de las necesidades sociales referente a los servicios que reciben, en pro de velar por el potencial humano que se encuentra en proceso de formación bajo nuestra responsabilidad.

- Es necesario centrar nuestra atención en la calidad de servicios del sistema administrativo de la facultad ya que los estudiantes manifiestan constante maltrato.

- La transformación en la educación de enfermería requiere de nuevos proceso para enfocar la formación desde una perspectiva más íntegra por lo que necesita de cambios en la estructura organizativa de los programas de formación, orientación del currículo a fin de lograr la tan ansiada Acreditación universitaria que permita ofrecer a la comunidad profesionales altamente competitivos, satisfechos con su formación académica.

\section{REFERENCIAS BIBLIOGRÁFICAS}

- Alberdi, R. Las enfermeras para el tercer milenio, rol de enfermería. México. pp 96. 1993.

- Cottle, D. El Servicio Centrado en el Cliente. 1991.

- Chaska, N.. La profesión de enfermería. pp.177. 1987

- Donabedian A. Gerencia para la calidad total en salud. 1990.
- James, Robinson. 1991. Calidad de atención en salud. México: ENSAP, pp 133.

- Laferriere, R. J. Client satisfaction with House Health Care Nursing. Community-Health-Nurse. pp 67-76. 1993.

- Leonard, Betty. y David Bennet. Calidad en servicio. pp. 124. 1998.

- Luverna, W. 1989. El pensamiento crítico en enfermería práctica. Segunda edición Barcelona: Mosby - Doyma. pp. 12 y 156.

- Petters, Tom. Tratado de la Calidad. Pp 501. 1994

- Sánchez, B. Identidad y empoderamiento de la profesión de enfermería. México: Interamericana. 2002.

- Salazar, G. Calidad y enfermería. México: Interamericana. pp 147. 2000.

- Salazar, M. Calidad total. Perú. Tercera Edición, Set S. R. L. 1995. 